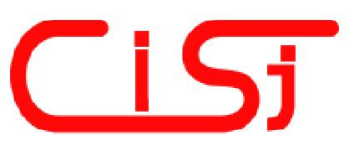

\title{
ADVANCED EXPERIMENTAL BIFURCATION ANALYSIS: BASELINE SCENARIO OF DYNAMICS EVOLUTION
}

\author{
Yury V. Kolokolov ${ }^{1)}$, Anna V. Monovskaya ${ }^{1,2)}$ \\ 1) RIHMI-WDC, Obninsk, Russia, www.meteo.ru \\ ${ }^{2)}$ ITMO University, Kronverkskiy 49, 197101, St. Petersburg, Russia, www.ifmo.ru \\ 2kolo@mail.ru, anna.monovskaya@mail.ru
}

\begin{abstract}
The paper develops the advanced experimental bifurcation analysis, where the extended data acquisition, the intelligent data processing, and the modified bifurcation diagrams are combined to get useful information for different specified purposes. In particular, here we focus on how to estimate experimentally the nearest limit of the operating process stability loss without expert opinions. So, we consider the baseline scenario of dynamics evolution, where the peculiarity of the estimation is connected with the possibility to determine the uncertainty zone about the corresponding bifurcation points. The algorithms of both data acquisition and data processing are illustrated. The procedures of the algorithms are founded on the methods of the experimental researches of different nonlinear phenomena under uncertainties. The presented results can be applied to engineering design. Copyright $(\mathrm{C}$ Research Institute for Intelligent Computer Systems, 2014. All rights reserved.
\end{abstract}

Keywords: nonlinear time series analysis; experimental bifurcation analysis; modified bifurcation diagrams; uncertainty zone; shadow bifurcations.

\section{INTRODUCTION}

The bifurcation analysis remains the main tool to study the regularities of nonlinear dynamics evolution with variation of parameters. The identification of a dynamical process with a certain parameters is the main procedure of the bifurcation analysis, where a periodicity is one of the basic characteristics. So, the methods of the bifurcation analysis relate to the methods of the time-andfrequency analysis. In particular, the results of the spectral analysis are traditionally used to illustrate the experimental bifurcation diagrams, for example $[1,2,3,4,5]$. But, the purposes of the bifurcation analysis are essentially wider and deeper, and the necessary fundamental researches of nonlinear dynamics can be extremely difficult. For example, the bifurcation analysis allows to determine the regularities of the bifurcation phenomena with variation of parameters, and allows to determine bifurcation phenomena without changes of a periodicity (C-bifurcations or, in other words, border-collision bifurcations), and allows to identify multistability, and allows to study the interrelation between nonlinear phenomena and noise, including regularities within the uncertainty zones, and so on.

At the same time, it is necessary to notice that the methods of the computational bifurcation analysis have essential differences in comparison with the methods of the experimental bifurcation analysis. For example, the main benefits of the computational way are connected with the heightened controllability of the simulations: it is possible to calculate a certain bifurcation point between two dynamical processes, and then it is possible to study the regularities of nonlinear dynamics with any initial conditions and with variation of any parameter within any range. To make experimental researches, it is necessary to spend comparatively more time and to use comparatively more equipments, and to apply comparatively more identification methods than to make similar computational researches. But the main benefits of the experimental way are connected with the heightened reliability of the results $[5,6,7]$ : it is possible to get more correct a bifurcation point location, and to get the dynamics evolution without destruction and distortion of intricate interrelations between nonlinear phenomena and noise, and so on. The paper is devoted to the development of the experimental bifurcation analysis in a practical application.

So, the following engineering problem is one of the most actual: how to provide the operating process stability? In other words, it means to determine the corresponding nearest bifurcation 
limit. Currently, the process of the data acquisition to build a bifurcation diagram seems to be a typical work, which could be implemented by an experienced engineer based on industrial microprocessor devices. But the analysis of the acquired data remains the expensive and nontrivial expert work. The unavoidable simplifications of any mathematical model suppose that the corresponding computer simulations can be used only as preliminary solutions [7, 8]. As a result, the bifurcation analysis is not practically used in engineering $[9,10,11,12]$, although if the data processing could be completely formalized, then it could have wide perspective. So, we focus hereafter on how to estimate experimentally the nearest limit of the operating process stability without the expert opinions. With this purpose the extended data acquisition (section 2), the intelligent data processing (section 3) are combined to get the necessary useful information presented by the modified bifurcation diagrams.

\section{EXTENDED DATA ASQUISITION}

Different kinds of the period doubling 1-2-4-... cascade form a lot of baseline scenarios of the dynamics evolution for the systems of different nature, for example $[1,13,14]$. In particular, it is typical for the different pulse systems of energy conversion $[5,8,15]$, where the operating process with the synchronization period $\left(T_{S}\right)$ is the first process within the cascade. Each next periodic process is described by the ratio $(m)$ of its periodicity to $T_{S}$-period, and is denoted as an " $m$ process". One of the experimental bifurcation diagrams of 1-2-4-...scenario is shown in Fig. 1a, where an amplification coefficient $(\alpha)$ is a bifurcation parameter. The time series of 1-, 2-, 4processes are illustrated in Figs. 1b,c,d. Any process differing from the operating one represents emergency of more or less extent. If the evolution is developed through C-bifurcations, then the quantitative changes in dynamics can occur extremely abruptly, and such behavior seems to be the most dangerous. Moreover, $m$-process can be realized by several variants, and a symbolical characteristic is used to distinguish these variants. For example, 2-process can be realized as $(1,0)(0)$ process (Fig. 1c), or as $(1,0)(1,0)$-process, or as (1)(1,0)-process, for example [5]. The numerals 0 and 1 mean open and closed key element correspondingly, switches within $T_{S}$-period are restricted by the parenthesis. Here and after we use the results made on the experimental setup of DCDC drive with pulse-width modulation, detailed description of which is presented in $[5,8]$. Let us make several necessary brief comments.

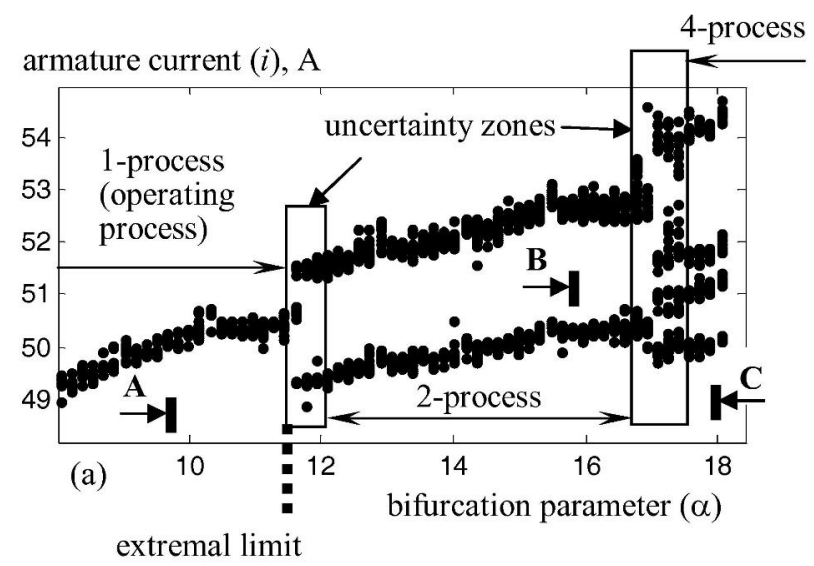

of the operating process stability

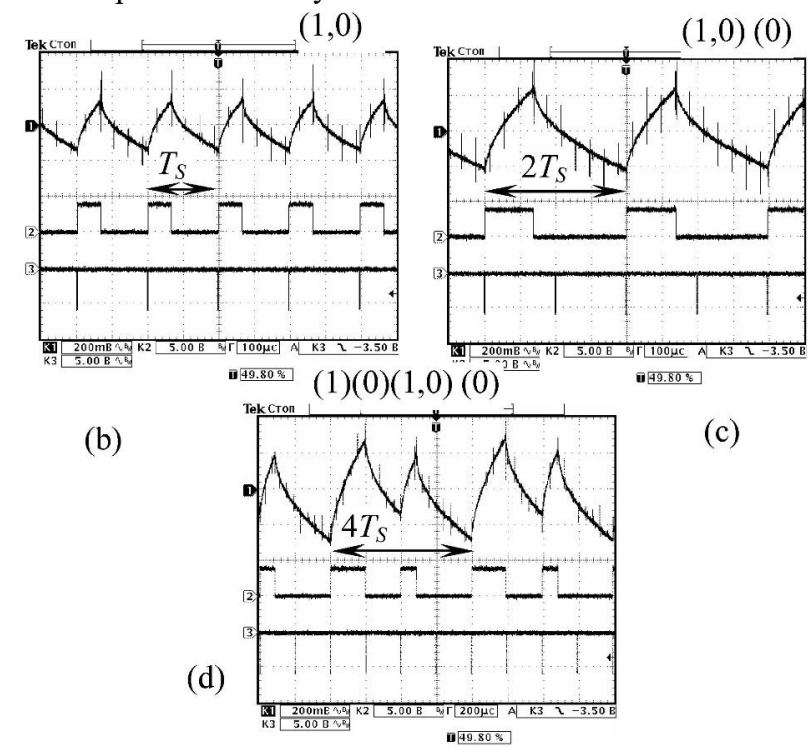

$1^{\text {st }}$ channel (upper) - the armature current; $2^{\mathrm{d}}$ channel the PWM voltage; $3^{\mathrm{d}}$ channel - the voltage of the synchronization pulse.

Fig. 1 - A traditional experimental bifurcation diagram with $n=200 \mathrm{rpm}$ (a); oscillograms of 1-, 2-, 4processes (b, c, d, correspondingly) made for A-, B-, C-sections in Fig. 1a.

An electromechanical part of the setup consists of two industrial $2 \mathrm{~kW}$ traction DC motors, one of which operates at the start mode. The second DC motor operates at the generator mode and represents a load for the first DC motor. So, the load is estimated by the rotation frequency $(n)$, where $n$ variation depends on the current $\left(I_{G R E F}\right)$ controlled by the source of a generator excitation current. The digital codes of $T_{S^{-}}, \alpha-, I_{G R E F^{-}}$-values are transferred by LPT-interface to the digital-analog converters of the control system. The digital oscilloscope is used to make the visual observations. One of the data acquisition systems contains 14-bit analog-digital converter, and is characterized by the sampling frequency of $10 \mathrm{MHz}$ per 4 channels, where data are transferred by USB 2.0 interface. It provides the 


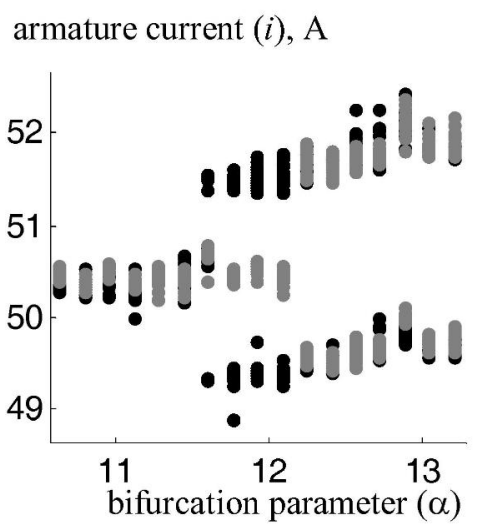

(a) armature current $(i), \mathrm{A}$

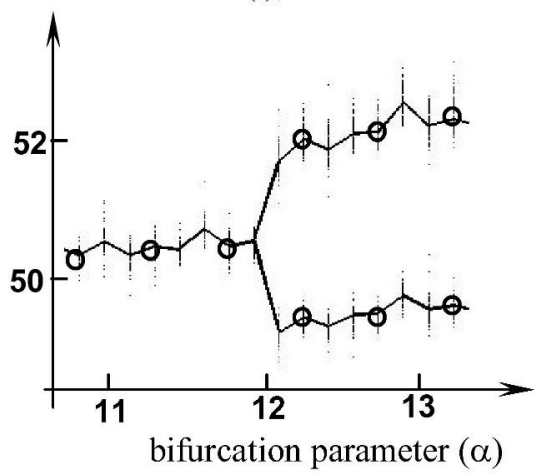

(b) armature current $(i), \mathrm{A}$

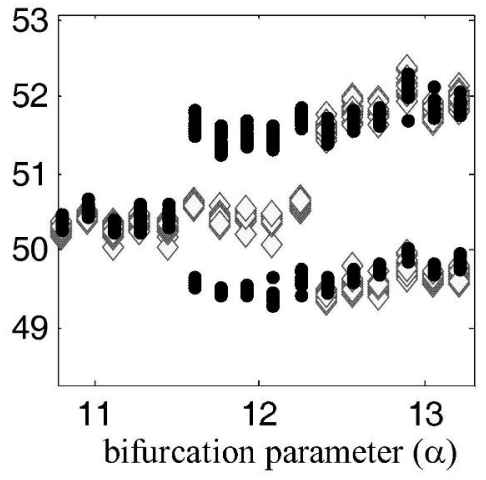

(c)

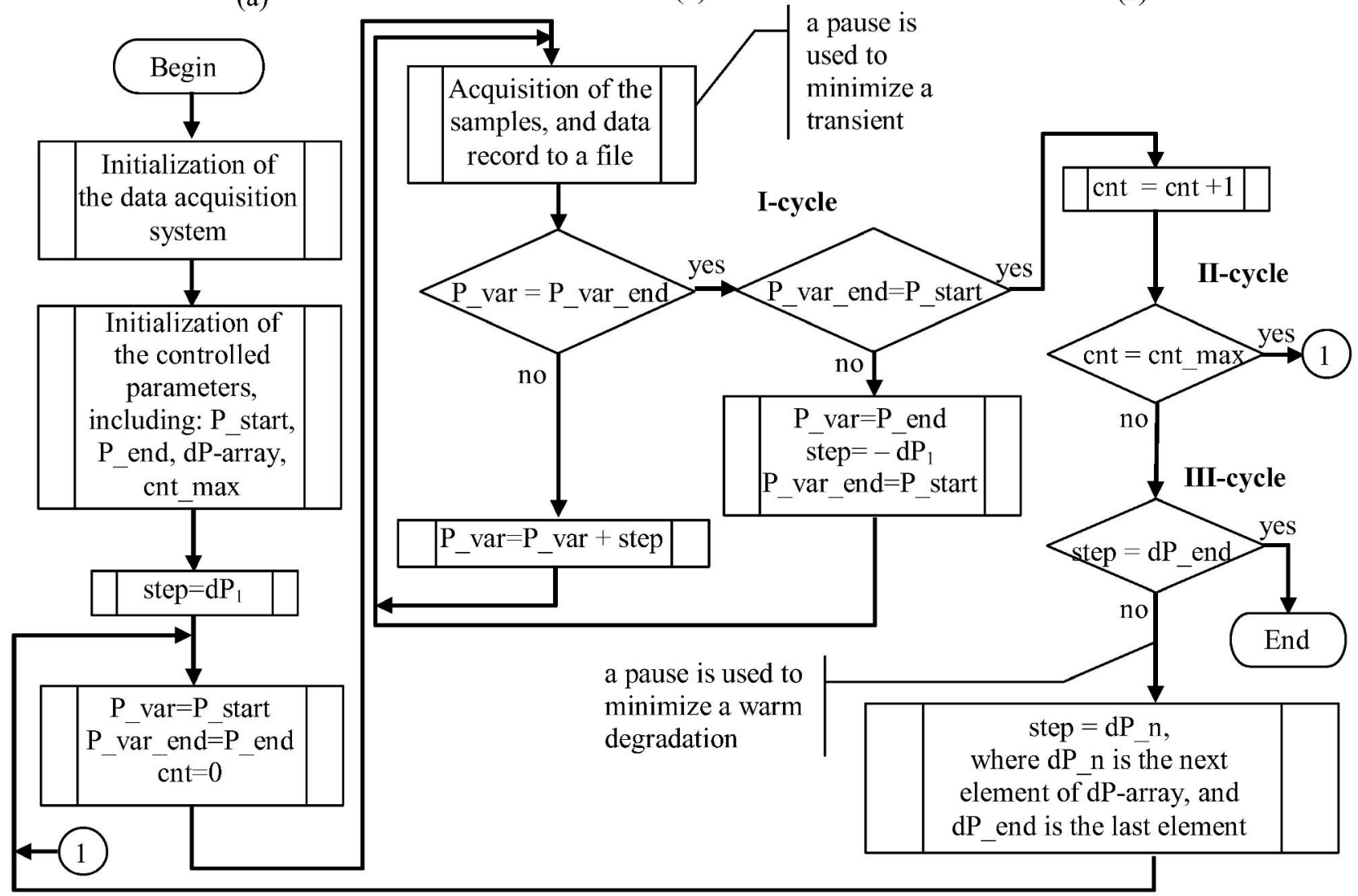

(d)

Fig. 2. - Effects about the 1,2-process bifurcation point, $\mathbf{n}=\mathbf{2 0 0} \mathbf{~ r p m}$ : the hysteresis effect, where black points correspond to $\alpha$-decrease, the gray points correspond to $\alpha$-increase (a); the effect of a step, where the solid lines correspond to $M$-values determined for the minimal step of $\alpha$-variation, the circles correspond to M-values determined for the threefold step of $\alpha$-variation (b); the warming degradation effect, where the black points correspond to $\alpha$-variation during the $1^{\text {st }}$ acquisition cycle, the rhombic points correspond to the $5^{\text {th }}$ acquisition cycle (c); the main procedures of the algorithm to the extended data acquisition (d).

incessant piping of the following data to PC: the armature current $(i)$ and $n$-samples (from the power part); the PWM-voltage and the synchronization pulse (from the control part). The acquired data are saved to a hard disk of PC. The samples can be formed with the preset frequency as well as "on the event" (a front/drop of a pulse function, front of a synchronizing clock, and so on). In particular we acquire $100 i$-samples per $T_{S}$-period to analyse the dynamics evolution in more details (see below
Figs. $3 \mathrm{c}, \mathrm{d})$. At the same time, one $i$-sample per $T_{S^{-}}$ period is traditionally acquired to build an experimental bifurcation diagram $[1,2,3,4,5]$, where the ratio $m$ is determined on the basis of the Poincaré's periodicity condition. Let us consider several moments which seem to be important to consider the allocation of a bifurcation point as comprehensive as possible.

The main drift of the bifurcation point is stipulated by the parametrical variation. But there 
are, at least, three effects which are usually irremovable and can lead to additional significant results in an electromechanical system of energy conversion. Both a direction (in more extent) and a step (in less extent) of parameter changes influence on where a bifurcation is realized. The corresponding examples are shown in Fig. 2a,b. The results of the computer simulations [16] show that the accumulated heat degradations of the elements can be noticeable, so we first introduce the corresponding cycle within the data acquisition to build the additional bifurcation diagrams. In particular, it allows to demonstrate the following: the effects of the heat degradations (Fig. 2c) can be comparable with the effects of the hysteresis drifts (Fig. 2a). Let us announce the extended data acquisition which allows to take into account the mentioned effects. Fig. $2 \mathrm{~d}$ shows the main procedures to implement it under the variation of one of the controlled parameter $(\mathrm{P})$ within the range [Pstart; Pend] with variable step $\left(\mathrm{dP}_{\mathrm{i}}\right)$, where the number of the iterations (cnt_max) is preset to imitate the heat degradations. The I-cycle realizes the P-variation to determine the bifurcation drift in cause of the change of the direction, where a pause is used after each P-value change to minimize a transient. The acquired $i$-samples are transferred from the data acquisition system to the $\mathrm{PC}$ and are written to a file. The II-cycle realizes the P-variation to determine the bifurcation drift in cause of the heat degradations. The III-cycle realizes the P-variation to determine the bifurcation drift in cause of the changes of the step, where a pause is used after each change to minimize the heat effects. Each bifurcation diagram built with a certain combination of $P_{-}, d P_{-}$, cnt-parameters is denoted a bifurcation realization (for example, Figs. 1a, 3a).

\section{INTELLIGENT DATA PROCESSING}

As a result of the extended acquisition the data volume becomes wider in thousands and more times, than it is usually supposed by the traditional experimental analysis. The developed data processing has the following aims: to exclude the subjective expert opinion, but to reveal more regularities; to analyse the widen data volume, but to reduce the duration of the analysis. Let us illustrate the corresponding specific methods used to build a bifurcation diagram as correct as possible. At present there are methods of real-time identification of the direction of the running transient convergence known for pulse systems [7], which are based on the use of the symbolical characteristics of $m$-processes. And it is possible to show that such methods provide one of the best tools to distinguish the operating process from other ones by analysis of time series under uncertainties of different nature [5]. In particular, one of such methods is based on the calculation of $m, r$-symbolical characteristics. In this case each bifurcation branch is identified in relation to the previous one. At the first step, the branch of operating process (Fig. 1b) is identified while 1,0characteristic remains. When the symbolical characteristic becomes equal to 2,0-characteristic, the $(1,0)(0)$-process (Fig. 2c) appears. For example,

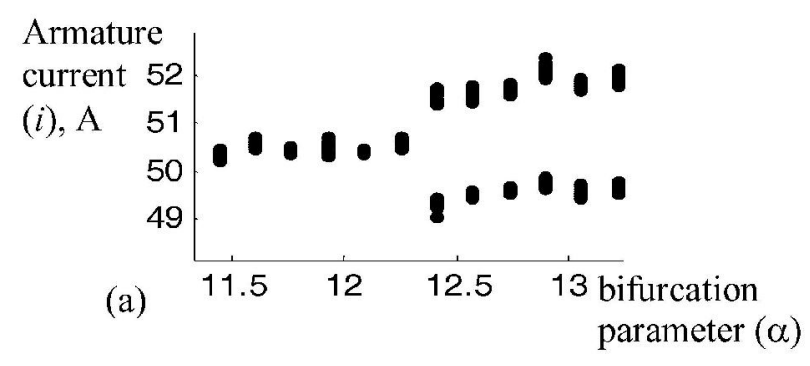

$m, r$-symbolical characteristics
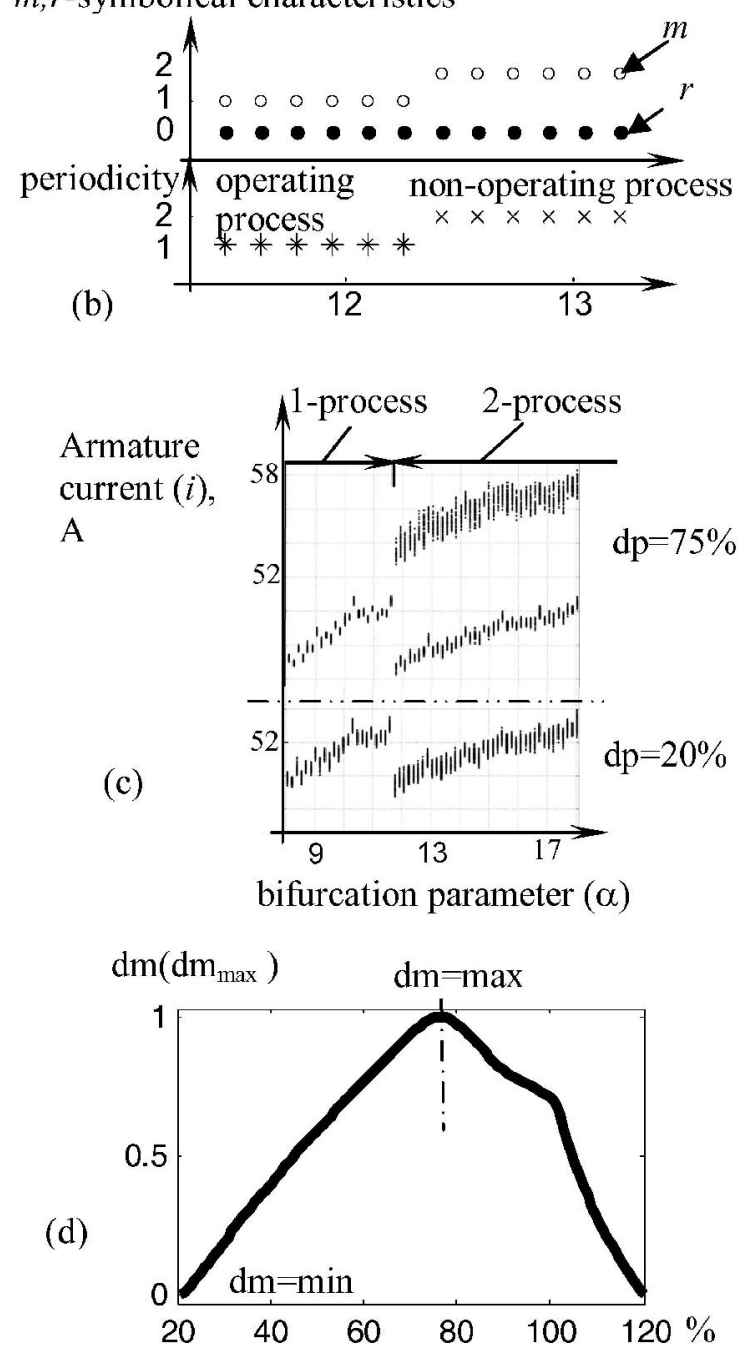

Fig. 3 - The fragment of one bifurcation realization about 1,2-process bifurcation point (a) with the synchronized time series to exhibit the result of the dynamics identification (b); the views of the bifurcation realization with $\mathrm{dp}=\mathbf{7 5 \%}$ and $\mathrm{dp}=\mathbf{2 0 \%}(\mathrm{c}$, upper and low parts) and the $\mathrm{dm} / \mathrm{dm}_{\max }(\mathrm{dp})-$ dependence (d). 
the results of the identification are presented in Figs. 3a,b. At the second step, each of two branches of $(1,0)(0)$-process has 1,0-characteristic, and the branches of $(1)(0)(1,0)(0)$-process (Fig. $2 \mathrm{~d}$ ) are divided: the up pair of the branches has 2,1characteristic; the low pair of the branches has 2,0characteristic. So, it is possible to identify step-bystep the bifurcation points within a bifurcation realization [5].

The next specific procedure is connected with the use of the method to tune the Poncare's mapping to avoid the shadow bifurcations and to get the optimized view of each realization [6,7]. What does it mean? Let $d p$ is a relative distance of the first point of the Poincare mapping (first $i$-samples) from the begin of PWM period (when $i$-value begin to increase). Then, the view of each bifurcation realization can change with $d p$-variation. For example, the view of the bifurcation realization with $\mathrm{dp}=75 \%$ (Fig. 3c, upper part) is characterized by the maximal distance between the 2-process branches, that allows to consider it as the optimal one. At the same time, the view of the bifurcation realization with $\mathrm{dp}=20 \%$ (Fig. 3c, low part) is characterized by the minimal distance between the 2-process branches, that looks like 1-process branch and can lead to identification of the shadow bifurcation with incorrect bifurcation parameter [6]. The $d p$-variation from $0 \%$ to $100 \%$ is illustrated in Fig. 3d. Such dependence is built only for several bifurcation realizations to determine the averaged maximal $d p$ value in order to tune the presentation. In other words, in contrast to the previous fundamental researches $[5,6,7]$, it is not necessary to reveal the novel nonlinear phenomena and to reveal the novel regularities, it is enough only to use and to tune the known general rules.

The mentioned reasonings allow to announce the corresponding algorithm of the intelligent data processing, main procedures of which are presented in Fig. 4a. The algorithm is generally founded on the procedures used by the methods to build the modified bifurcation diagrams [6], and is adapted to a certain purpose. In particular, the purpose is to estimate the nearest limit of the operating process stability without the expert opinions. To realize the purpose the following flow are minimal and defensible to get the necessary information. First, a binary file is created for each bifurcation realization as a result of the data acquisition (Fig. 2d), so it is necessary to converse all the binary files to the corresponding mat-files. Next, it is necessary to identify a bifurcation point for each bifurcation realization by the cycle, at the end of which the array containing the corresponding results is filled. In particular, the method based on the calculation of $m, r$-symbolical characteristics (Figs. 3a,b) is used to realize the identification.

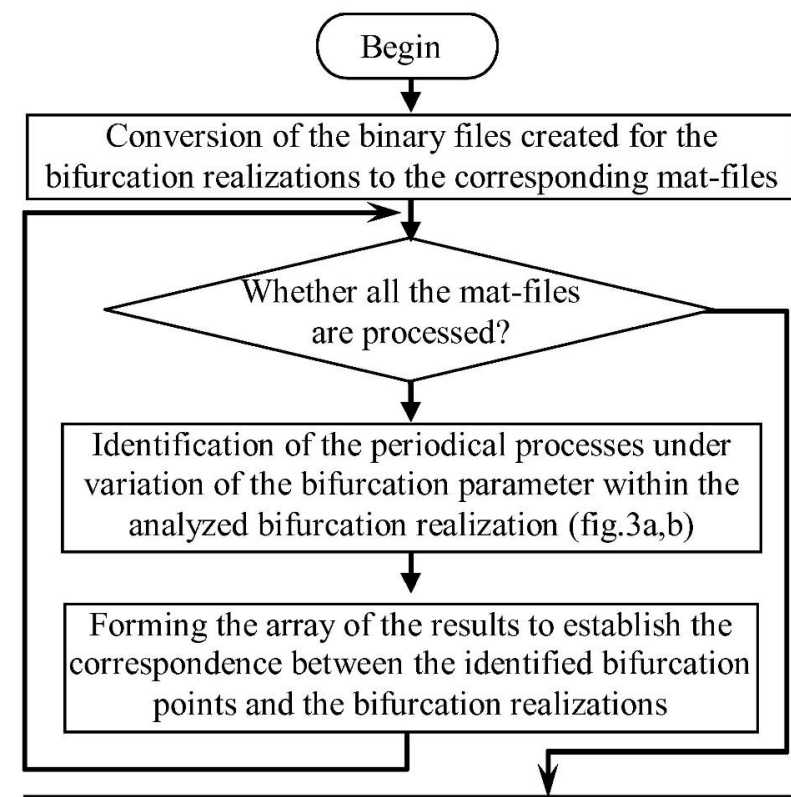

Tuning the Poincare's mapping to avoid the shadow bifurcation points and to get the optimized view of each experimental bifurcation diagram (fig. $3 \mathrm{c}, \mathrm{d}$ )

Analysis of the formed array: to choose the mat-files, where the bifurcation realizations have the minimal and the maximal values of the bifurcation points. Forming the samplings with $d p$-value optimized to visualize the bifurcation points.

Building the modified bifurcation diagrams to show the operating zone, the uncertainty zone, and the nonoperating zone (fig.4b)

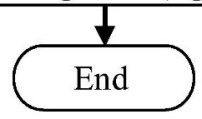

(a)

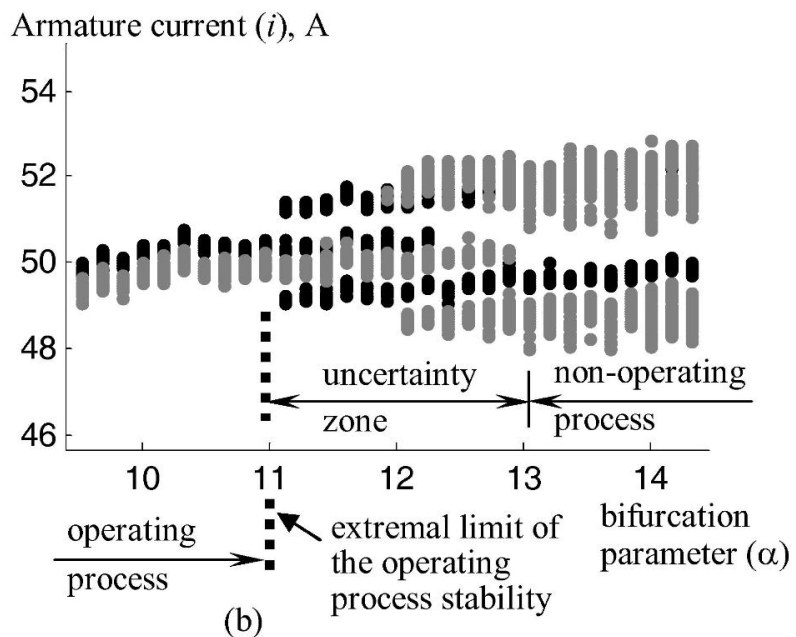

Fig. 4. - The main procedures of the algorithm of the intelligent data processing (a); example of the extremal limit of the operating process stability (b) based on the combination of the results made for one series of the experiments with $n=200 \mathbf{r p m}$ (black poins) and one series of the experiments with $n=400$ rpm (gray points). 
Third, it is necessary to analyse the array in order to determine the extremal limit of the operating process stability, and to build the corresponding modified bifurcation diagram to demonstrate the limits of the operating process, the uncertainty zone, and the nonoperating zone. An example to illustrate the modified bifurcation diagram is presented in Fig. 4b. Such diagram is essentially simplified variant of the known modified bifurcation diagrams $[5,6,7]$, which are used to reveal the regularities of each of the effects observed within the ensemble of the nonlinear phenomena about bifurcation points. In other words, the presented diagram illustrates mainly the maximal drift of the first bifurcation point without the specifications on the particular effects.

\section{CONCLUSION}

So, the extended data acquisition allows to get essentially more ordered data concerning the reactions of the dynamical system on the combinations of both the preset changes of the controlled parameters and uncertainties. The proposed algorithm of the intelligent data processing represents the tool to analyse the widen data volume, but to reduce the duration of the analysis, where the subjective expert opinion is excluded, but the extremal limit of the operating process stability is determined and demonstrated. Here we first propose how the experience accumulated while experimental researches of the complex ensembles of nonlinear phenomena about bifurcation points could be adapted to engineering design. We believe that such simplified adaptation seems to be the most promising to improve the reliability of the responsible parts of technological processes. Also, we believe that the algorithm can be used as the framework for the wide class of the researches connected with the following topics: to study the operating process domain; to study the uncertainty zones about bifurcation points; to study nonoperating process domains. Here it is necessary to notice, that because of different kinds of the considered period doubling 1-2-4-... cascade form a lot of baseline scenarios of the dynamics evolution for the systems of different nature, then the presented algorithms could be interesting to be adapted for other nonlinear dynamics systems.

\section{REFERENCES}

[1] P. Berge, Y. Pomeau, Ch. Vidal, Order Within Chaos: Towards a Deterministic Approach to Turbulence, NY: John Wiley \& Sons, New York, 1984, 329 p.

[2] J. A. Glazier, and A. Libchaber, Quasiperiodicity and dynamical systems: an experimentalist's view, IEEE Trans. Circuits and Systems, (35) 7 (1988), pp. 790-809.

[3] E. Sanchez, D. Pazoa, M. A. Matias, Experimental study of the transitions between synchronous chaos and a periodic rotating wave, Chaos, (16) (2006), 033122.

[4] E. Bureau, F. Schilder, I. F. Santos, J.J. Thomsen, J. Starke, Experimental bifurcation analysis of an impact oscillator tuning a non-invasive control scheme, $J$. of Sound and Vibration, (322) 22 (2013), pp. 5883-5897.

[5] Yu. V. Kolokolov, A. V. Monovskaya, Estimating the uncertainty of the behavior of a PWM power converter by analyzing a set of experimental bifurcation diagrams, Int. J. of Bifurcation and Chaos, (23) 4 (2013), 1350063.

[6] Yu. V. Kolokolov, A. V. Monovskaya, From modifications of experimental bifurcation diagrams to operating process stability margin, Int. J. of Bifurcation and Chaos, (23) 7 (2013), 1330024.

[7] Yu. V. Kolokolov, A. V. Monovskaya, Fractal approach, bifurcation poker and SUC-logic for nonlinear dynamics forecasting, Int. J. of Bifurcation and Chaos, (23) 12 (2013), 1350201.

[8] Yu. V. Kolokolov, S. L. Koschinsky, V. V. Bagrov, Experimental dynamics of electromechanical pulse energy conversion system, IEEE Trans. on Instrumentation and Measurement, (55) 1 (2006), pp. 35-43.

[9] M. Gelali, An overview of control performance technology and industrial applications, Control Engineering Practice, (14) (2006), pp.441-466.

[10] L. M. C. Medina, R. de J. Romero-Troncoso, E. Cabal-Yepez, J. de J. Rangel-Magdaleno, J. R. Millan-Almaraz, FPGA-based multiplechannel vibration analyzer for industrial applications in induction motor failure detection, IEEE Trans. on Instrumentation and Measurement, (59) 1 (2010), pp. 63-72.

[11] A. Gandhi, T. Corrigan, L. Parsa, Recent advances in modeling and online detection of stator interturn faults in electrical motors. (review), IEEE Trans. on Industrial Electronics, (58) 5 (2011), pp. 1564-1575.

[12] C.-H. Huang, C.-H. Lee, K.-J. Shih, Y.-J. Wang, A robust technique for frequency estimation of distorted signals in power systems, IEEE Trans. on Instrumentation and Measurement, (59) 8 (2010), pp. 2026-2036.

[13] V.-Th. Pham, A. Buscarino, L. Fortuna, M. Frasca, Simple memristive time-delay chaotic systems, Int. J. of Bifurcation and Chaos, (23) 4 (2013), 1350073. 
[14] E. Sander, J. A. Yorke, Connecting perioddoubling cascades to chaos, Int. J. of Bifurcation and Chaos, (22) 2 (2012), 1250022.

[15] Yu. V. Kolokolov, S. L. Koschinsky, C. Hatziadoniu, G. Galanos, Dynamics of current regulator with hysteresis control and clocked commutation in application to power electronic systems, 2003 2d IEEE Int. Conference on Physics and Control, St. Petersburg, Russia (August 20-23, 2003), pp. 1165-1169.

[16] Yu. V. Kolokolov, A. V. Monovskaya, An influence of parameter variation on modelbased emergency forecasting in the pulse system, in Proceedings of the 2nd IEEE Workshop on Intelligent Data Acquisition and Advanced Computer Systems: Technology and Applications, Lviv, Ukraine (September 8-10, 2003), pp. 349-354.

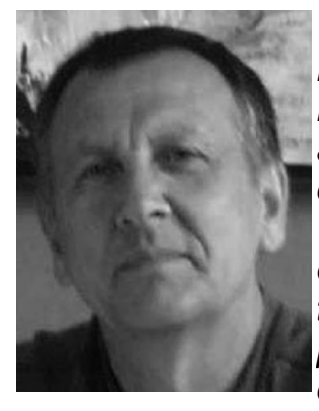

Yury V. Kolokolov received PhD degree in 1978 (Tomsk Politechnical Institute, Russia), and Russian academic degree of Senior Doctor in 1990 (Tomsk University of Control and Radioelectronics, Russia). From 1993 to 2007 he worked as a professor and Head of the Dept. of Design and technology of electronic systems (State Technical University of Orel, Russia), from 2007 to 2012 he worked as a professor and Director of Institute of Control Systems and Information Technologies (UGRA State University, Khanty-Mansiysk, Russia). He is currently a chief researcher (Russian Research Institute of Hydrometeorological Information - World Data Center, Obinsk, Russia). His research interests include theories and practice of nonlinear dynamics in complex technical and natural systems.

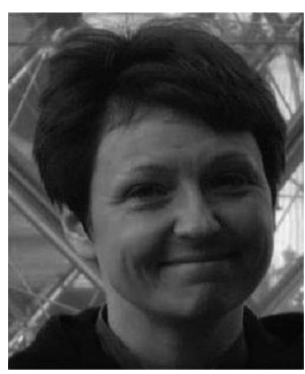

Anna V. Monovskaya received PhD degree in 2005 (State Technical University of Orel, Russia and University of Technology of Troyes, France), and Russian academic degree of Senior Doctor in 2010 (UGRA State University, Khanty-Mansiysk, Russia). From 2007 to 2012

she worked as a professor and Head of the Dept. of Automated systems of data processing and control (UGRA State University, Khanty-Mansiysk, Russia). She is currently a chief researcher (Russian Research Institute of Hydrometeorological Information - World Data Center, Obinsk, Russia and ITMO University, St. Petersburg, Russia). Her research interests include theories and practice of nonlinear dynamics in complex technical and natural systems. 